\title{
Comparação da resposta autonômica cardiovascular de praticantes de musculação, corredores de longa distância e não praticantes de exercício
}

CDD. 20.ed. 796.022 796.073

\author{
Wellington LUNZ \\ Rodrigo Nascimento MIRANDA* \\ Eduardo Miranda DANTAS ${ }^{* *}$ \\ Elis Aguiar dos Santos MORRA* \\ Luciana CARLETTI* \\ Anselmo José PEREZ* \\ José Geraldo MILL*
}

*Centro de Educação Física e Desportos, Universidade Federal do Espirito Santo. **Universidade Federal do Vale do São Francisco.

\section{Resumo}

Objetivou-se comparar a resposta autonômica cardiovascular (RAC) de praticantes de musculação, corredores de longa distância e não praticantes de exercício. Homens, 21 a 55 anos, foram agrupados em: Praticantes Musculação (PM, $n=31)$; Praticantes Corrida ( $P C, n=28)$; Controle $(C, n=35)$. Foram selecionadas quatro técnicas para avaliação da RAC: Frequência cardiaca de repouso (FCR), Teste pressórico do frio (TPF), Variabilidade da FC (VFC) e recuperação da FC pós-teste máximo em esteira. $A$ FCR foi menor no grupo $P C(P C=54 \pm 2 ; P M=62 \pm 2 ; C=65 \pm 2$ bpm; média $\pm E P M)$. A recuperação da $F C$ aos 60 s pós-teste de esforço foi maior no grupo $P C(P C=34 \pm 3 ; P M=23 \pm 1 ; C=24 \pm 2 ;$ bpm $)$. Quanto aos parâmetros espectrais de alta (HF) e baixa (LF) frequência da VFC, o grupo PC apresentou maior $H F(55,1 \pm 4,0$ n.u) e menor $\operatorname{LF}(43,1 \pm 4,0$ n.u) comparado ao grupo $C(H F=40,7 \pm 3,3 ; L F=56,7$ $\pm 3,5$ n.u). 0 grupo PM não apresentou qualquer diferença de RAC em comparação ao grupo C. Concluise que prática contínua em musculação por longo prazo, diferentemente da prática de corrida de longa distância, não é capaz de alterar significativamente a RAC.

PalavaAs-chave: Treinamento de força; Exercício aeróbio; Sistema nervoso simpático; Sistema nervoso parassimpático; Controle autonômico do coração; Variabilidade da frequência cardíaca.

\section{Introdução}

O sistema nervoso (SN) autônomo (SNA) é responsável por várias funções homeostáticas, incluindo modulação da pressão arterial (PA) e frequência cardíaca $(\mathrm{FC})^{1-3}$.

O interesse clínico pelo comportamento do SNA tem aumentado porque vários estudos têm mostrado que alteraçōes autonômicas crônicas estão associadas ao desenvolvimento de doenças cardiovasculares e aumento do risco de mortes ${ }^{4-5}$. De fato, o aumento da FC de repouso (FCR) em aproximadamente 15 batimentos, num intervalo de 10 anos, foi associado positivamente com o risco de mortes por doença cardíaca isquêmica ${ }^{6}$. Resultados concordantes já haviam sido descritos anteriormente ${ }^{3,7}$. Ressalta-se que um aumento significativo da FCR é sugestivo de atividade diminuída e aumentada dos componentes parassimpático e simpático, respectivamente, do $\mathrm{SNA}^{2-3,35-6,8}$.

A recuperação mais lenta da FC pós-teste de esforço, a qual é sugestiva de reativação lenta do componente parassimpático ${ }^{9-11}$, também prediz mortes por todas as causas e por doenças cardíacas ${ }^{3,10}$. A diminuição da capacidade de variação da FC pelo SNA também está associada com doenças cardiovasculares, como insuficiência cardíaca e infarto ${ }^{12-14}$.

A forma direta de avaliação da capacidade modulatória do SNA sobre o aparelho cardiovascular é de difícil 
aplicação no contexto clínico, pois exige o registro direto, de forma invasiva, da atividade elétrica de fibras nervosas superficiais ${ }^{3}$. Por outro lado, as formas indiretas são viáveis no âmbito clínico e epidemiológico ${ }^{3}$. Nesse contexto, a FC e PA, pela facilidade de obtenção de seus registros, são frequentemente utilizadas por quatro estratégias diferentes, mas complementares, que se destacam pela objetividade e viabilidade clínica: FCR, recuperação da FC após esforço físico, variabilidade da frequência cardíaca (VFC), e o comportamento da PA no teste pressórico do frio (TPF) ${ }^{3,5-6,11,15}$.

Por sua vez, ações modulatórias do SNA frente a alteraçōes orgânicas induzidas por sessões agudas de exercício são bem conhecidas ${ }^{1-2}$. Também é bem conhecido que o treinamento de característica aeróbia

\section{Método}

\section{Caracterização do estudo e conduta ética}

Trata-se de estudo observacional com delineamento transversal em amostra de voluntários. Os participantes assinaram "Termo de Consentimento Livre e Esclarecido" e a condução do estudo respeitou a Resolução 196/96 do CNS. O estudo foi aprovado no Comitê de Ética do Centro de Ciências da Saúde - UFES (protocolo 009/2010).

\section{Amostra}

Foram incluídos 94 homens, com qualquer grau de escolaridade, classificação étnica e socioeconômi$\mathrm{ca}$, com idade entre 21 e 55 anos. $\mathrm{O}$ recrutamento foi feito em academias de ginástica de Vitória - ES e em grupos de corredores que treinavam para participação em corridas longa distância (ex: maratona e meia maratona), e em pelo menos quatro importantes eventos de corrida do calendário capixaba. Praticantes com no mínimo dois anos de prática regular nas modalidades musculação e corridas foram divididos em dois grupos: 1) Praticantes de musculação (PM; $n=31$ ), caracterizado por treinamento de hipertrofia ou força máxima; 2) Praticantes de corrida de longa distância (PC; $n=28)$, caracterizado por treinamento superior a $40 \mathrm{~km} /$ sem, e frequência maior ou igual a três sessões/sem. Foi também incluído um grupo controle $(\mathrm{C} ; n=$ 35), composto por homens aparentemente saudáveis que não haviam se engajado em qualquer programa regular de treinamento nos últimos seis meses. de médio e longo prazo é capaz de modular o SNA, em particular o tônus parassimpático ${ }^{5,8,10-11,16-17}$.

Entretanto, com exceção de alguns poucos estudos de curta duração que avaliaram o efeito do treinamento de força sobre o SNA em modelo animal ${ }^{18}$ ou humanos ${ }^{12,17,19-21}$, ainda não está estabelecido se a prática de treinamento de força de longo prazo é capaz de modular o SNA.

Dessa forma, o principal objetivo do presente estudo foi comparar a resposta autonômica cardiovascular de praticantes de musculação, corredores de longa distância e não praticantes de exercício. Para uma melhor compreensão do fenômeno, as respostas do SNA foram avaliadas por quatro técnicas não invasivas e aplicáveis em contexto clínico.

Após entrevista para triagem feita pelos pesquisadores, agendava-se um dia para comparecimento à Clínica de Investigação Cardiovascular da UFES para realização de exames. Os procedimentos incluíam avaliação do risco cardiovascular e antropométrica, aferição da PA e FC, registros do ECG, ergoespirometria e testes para avaliação do SNA. Aos participantes solicitou-se comparecimento pela manhã, em jejum de $12 \mathrm{~h}$, e com abstenção de atividade física no dia anterior. $\mathrm{O}$ teste ergoespirométrico foi agendado em outro dia e horário, pois era realizado em outro espaço físico, no Laboratório de Fisiologia do Exercício do Centro de Educação Física e Desportos da UFES. Não foram incluídos no estudo os que relataram ser tabagistas ou consumidores regulares de bebidas alcoólicas (consumo médio superior $30 \mathrm{~g}$ de álcool/dia), ter história de morte súbita em familiares de $1^{\circ}$ grau e doença cardíaca já conhecida.

\section{Medidas antropométricas}

Foram realizadas em jejum de 12 horas. Obtevese peso corporal e estatura para posterior cálculo do IMC $\left(\mathrm{kg} / \mathrm{m}^{2}\right)$, dobras cutâneas triciptal e abdominal (Plicômetro Mitutoyo/CESCORF - 0,1 mm) e perimetrias do braço e perna (Fita flexível, Sanny). A perimetria do braço foi obtida após contração muscular dos flexores do cotovelo, com cotovelo e ombro flexionados em $90^{\circ}$. A perimetria da perna foi obtida após flexão plantar. As perimetrias foram feitas no maior perímetro do segmento direito. 


\section{Pressão arterial (PA) e frequência cardíaca (FC) de repouso (FCR)}

Foram obtidas três medidas da PA e FC em jejum e após repouso na posição sentada por pelo menos cinco minutos, em sala silenciosa e com temperatura controlada $\left(22-24^{\circ} \mathrm{C}\right)$. As medidas foram feitas em aparelho oscilométrico (Omron ${ }^{\circledR} 705 \mathrm{CP}$, Intelissense, Japão) validado ${ }^{22}$. O valor médio das três medidas foi considerado na análise dos dados. Após a antropometria e medidas da PA e FCR, os participantes fizeram o desjejum no próprio local dos exames e, após $30 \mathrm{~min}$ de intervalo, deu-se prosseguimento aos demais exames.

\section{Teste pressórico do frio (TPF)}

Após 5 min acomodados em cadeira, aferiu-se a PA (Omron $\left.{ }^{\circledR} 705-\mathrm{CP}\right)$ no braço direito (medida de repouso). Em seguida os sujeitos foram orientados que quando demandados pelo aferidor deveriam mergulhar a mão direita em água gelada $\left(4^{\circ} \mathrm{C}\right)$, e informados que a retirada da mão só deveria ocorrer após nova ordem. Foram alertados quanto à possível sensação de dor e desconforto que seriam gerados pelo teste. Não era informado ao participante o tempo de duração do teste. Após isso, aferia-se novamente a PA (resposta antecipatória). Na sequência, o participante submergia a mão até o punho na água gelada. Ao final do teste que durava $2 \mathrm{~min}$, mas anteriormente a retirada da mão, mensurava-se novamente a PA (resposta ao frio). Após a retirada da mão, com o participante ainda sentado, aguardavase mais 2 min para aferir novamente a PA (resposta de recuperação). As aferições da PA foram feitas no braço contralateral ao braço testado.

\section{Eletrocardiograma (ECG) e análise da variabilidade de frequência cardíaca (VFC)}

Realizou-se registro contínuo (10 min) de ECG, em decúbito dorsal e em ambiente silencioso com temperatura controlada $\left(22-24^{\circ} \mathrm{C}\right)$. Os registros foram obtidos com eletrocardiógrafo digital (Micromed, taxa de amostragem: $250 \mathrm{~Hz}$ ), e software específico (Wincardio 4.4a) que gerava as séries de intervalos R-R a partir da derivação de maior amplitude da onda R (geralmente DII). As análises de VFC foram realizadas no domínio do tempo e da frequência usando "software" personalizado em Matlab, conforme DANTAS et al. ${ }^{23}$. As séries foram pré-processadas para remoção de batimentos ectópicos e artefatos. As análises no domínio do tempo incluíram a raiz quadrada da média da soma dos quadrados das diferenças entre intervalos R-R adjacentes (rMSSD) e a razão obtida entre o número de pares adjacentes de intervalos R-R superiores a $50 \mathrm{~ms}$ pelo número total de intervalos $\mathrm{R}-\mathrm{R}$ da série ( $\mathrm{pNN} 50)$. A análise espectral foi realizada por modelamento autorregressivo usando método de Yule-Walker com algoritmo recursivo de LevisonDurbin $^{23}$. A ordem do modelo foi ajustada em todas as análises em 16. Os componentes oscilatórios presentes nas séries temporais foram classificados da seguinte forma: muito baixa frequência (VLF, $0-0,04 \mathrm{~Hz}$ ), baixa frequência (LF, 0,04-0,15 Hz), e alta frequência $(0,15-0,40 \mathrm{~Hz})$. Os valores dos índices espectrais foram apresentados em unidades normalizadas. A normalização consistiu em dividir a energia espectral de cada componente oscilatório pela energia total do espectro menos a energia do componente VLF. A razão LF/HF foi obtida pela divisão dos componentes LF pelo $\mathrm{HF}^{24-25}$.

\section{Teste de esforço máximo em esteira}

Os testes foram aplicados por profissionais experientes e com acompanhamento de um cardiologista. Utilizou-se esteira ergométrica (Inbrasport Super ATL) e um ergoespirômetro (Córtex, modelo Metamax 3B) para realização dos testes. Previamente ao teste obtinha-se registro de ECG e medida da PA. Utilizou-se protocolo de rampa, o qual progredia de cinco a $14 \mathrm{~km} / \mathrm{h}$ para o grupo PM, de seis a $12 \mathrm{~km} / \mathrm{h}$ para o grupo C e de seis a $22 \mathrm{~km} / \mathrm{h}$ para o grupo $\mathrm{PC}$. $\mathrm{O} \mathrm{VO}_{2 \max }$ era aceito quando os seguintes critérios eram contemplado ${ }^{26}$ : a) exaustão ou inabilidade para manter a velocidade requerida; b) razão de taxa respiratória superior a 1,15; c) FC máxima de pelo menos $90 \%$ da $\mathrm{FC}$ máxima estimada pela equação 220 - idade. A FC foi registrada pelo ECG durante todo o teste e acompanhada até o terceiro minuto da recuperação. Ao final do teste a velocidade era reduzida de cinco a $4 \mathrm{~km} / \mathrm{h}$ durante um minuto, seguido por mais um min de recuperação passiva.

\section{Análise estatística}

Os dados estão apresentados como "média \pm EPM, seguidos de mediana, que está apresentada dentro do parênteses. Dados com distribuição normal foram analisados com ANOVA de uma via 
seguido pelo "post-hoc" de Tukey. Os dados que não atenderam o pressuposto de normalidade foram analisados pelo teste equivalente não paramétrico de Kruskal-Wallis seguido pelo "post-hoc" de Dunn's. A normalidade foi avaliada pelo teste de Kolmogorov-Smirnov. Análise de Covariância (ANCOVA) de uma via foi utilizada quando a idade

\section{Resultados}

A caracterização da amostra está apresentada na TABELA 1. Os grupos PM e PC relaram exercitar continuamente por aproximadamente 10 anos. $\mathrm{O}$ grupo PC apresentou menor FCR e maiores valores para $\mathrm{VO}_{2 \max }$ e idade quando comparado aos demais grupos. $\mathrm{O}$ grupo PM apresentou valores superiores para peso corporal, IMC e perimetrias

TABELA 1 - Caracterização dos grupos estudados. se apresentou como covariável estatisticamente significativa. ANCOVA para medidas repetidas foi usada para comparar as medidas de PA e FC obtidas no TPF e teste cardiopulmonar, respectivamente. Os testes de ANCOVA foram seguidos pelo "post-hoc" de Bonferroni. A significância estatística, para todos os testes, foi estabelecida para $\mathrm{p}<0,05$.

em comparação aos grupos C e PC. O grupo $\mathrm{C}$ apresentou valor de dobra cutânea triciptal superior aos dois outros grupos, e de dobra cutânea abdominal superior ao grupo PC. A PA diastólica (PAD) medida antes do TPF (basal) foi menor no grupo PC. Não houve diferença entre os grupos para PA sistólica (PAS).

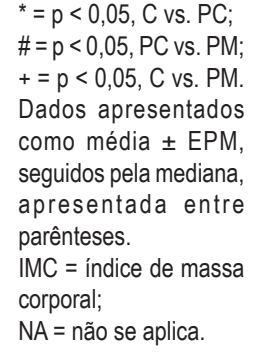

\section{Parâmetros}

\begin{tabular}{|c|c|c|c|}
\hline & $(\mathbf{n}=35)$ & $(\mathrm{n}=28)$ & $(\mathrm{n}=31)$ \\
\hline Idade (anos) & $32 \pm 1(30)^{*}$ & $38 \pm 2(38) \#$ & $32 \pm 1(30)$ \\
\hline Peso corporal (kg) & $75 \pm 2(72)+$ & $68 \pm 2(68) \#$ & $89 \pm 2(90)$ \\
\hline Estatura $(\mathrm{cm})$ & $176 \pm 1(176)$ & $173 \pm 1(172)$ & $177 \pm 2(175)$ \\
\hline $\operatorname{IMC}\left(\mathrm{kg} / \mathrm{m}^{2}\right)$ & $24 \pm 0,6(24)+$ & $23 \pm 0,4(22) \#$ & $28 \pm 0,7(28)$ \\
\hline Perimetria do braço $(\mathrm{cm})$ & $33 \pm 0,5(32)+$ & $31 \pm 0,4(31) \#$ & $42 \pm 0,6(42)$ \\
\hline Perimetria da perna $(\mathrm{cm})$ & $37 \pm 0,6(36)+$ & $37 \pm 0,5(36) \#$ & $40 \pm 0,5(40)$ \\
\hline Dobra cutânea triciptal (mm) & $10 \pm 0,6(9) *+$ & $6 \pm 0,4(6)$ & $6 \pm 0,4(6)$ \\
\hline Dobra cutânea abdominal (mm) & $30 \pm 2(29) *$ & $19 \pm 3(14)$ & $22 \pm 2(21)$ \\
\hline $\mathrm{VO}_{2 \max }\left(\mathrm{mL} \cdot \mathrm{kg}^{-1} \cdot \mathrm{min}^{-1}\right)$ & $39 \pm 1(39)^{*}$ & $51 \pm 1(49) \#$ & $41 \pm 1(40)$ \\
\hline Prática contínua em corrida de longa distância anos) & NA & $9,3 \pm 1,3(10)$ & NA \\
\hline Prática contínua em musculação (anos) & NA & NA & $10,7 \pm 1,0(10)$ \\
\hline Pressão Arterial Sistólica (mmHg) & $121 \pm 2(119)$ & $122 \pm 2(118)$ & $123 \pm 2(122)$ \\
\hline Pressão Arterial Diastólica (mmHg) & $74 \pm 1(72)^{*}$ & $71 \pm 1(70) \#$ & $74 \pm 1(72)$ \\
\hline Frequência Cardíaca de Repouso (bpm) & $65 \pm 2(64) *$ & $54 \pm 2(54) \#$ & $62 \pm 2(62)$ \\
\hline
\end{tabular}

As FIGURAS 1 e 2 mostram a variação da PAS e PAD no TPF, respectivamente. Com exceção ao grupo PC que apresentou PAD, antes do TPF, levemente inferior aos demais grupos, não houve diferenças significativas entre os grupos para os diferentes momentos do TPF. Nas comparações intra-grupo, como já era esperado, a resposta pressórica ao frio foi maior quando comparada aos momentos basal, antecipatório e recuperação (FIGURAS 1 e 2), evidenciando a participação do SNA simpático. A recuperação da PAS no grupo PM se manteve ligeiramente maior que a resposta basal (FIGURA 1). 


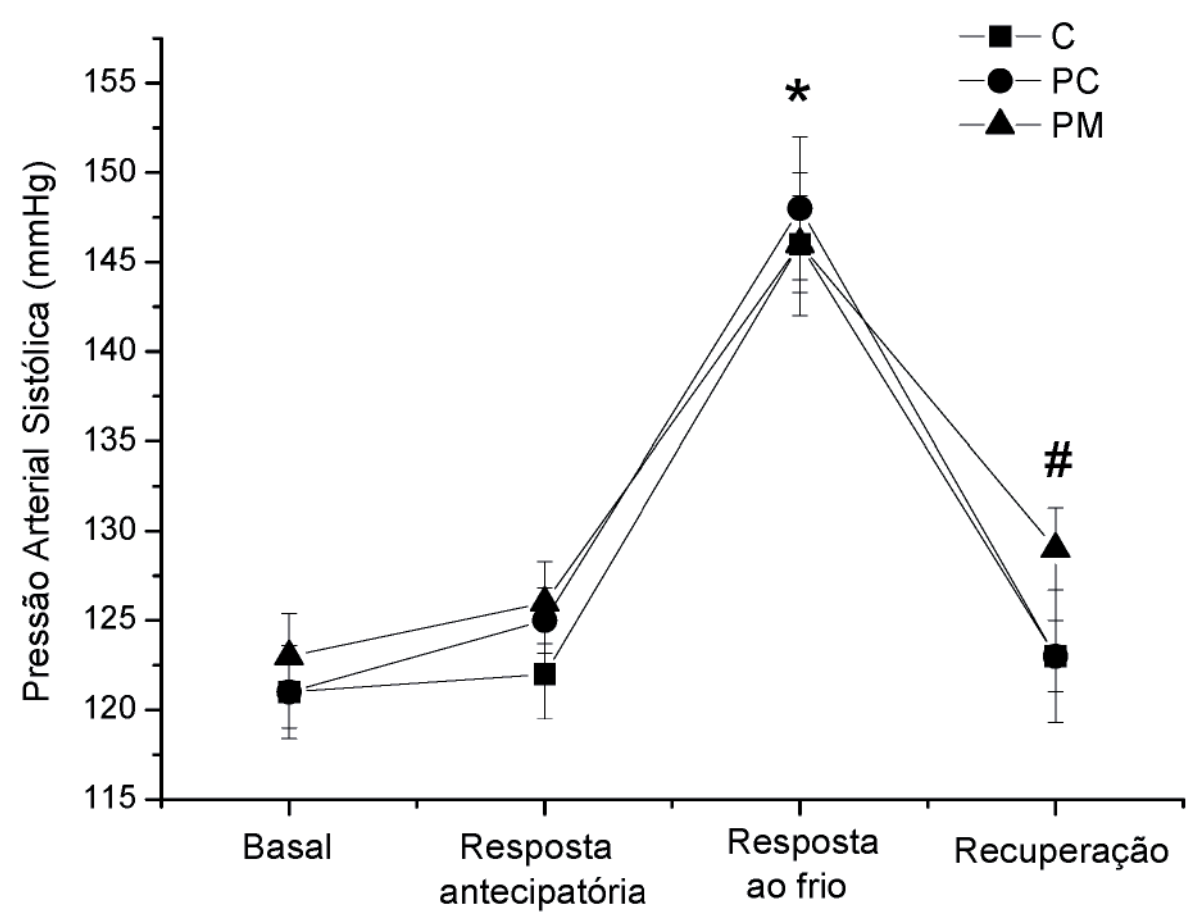

$*=p<0,05$, resposta ao frio vs. basal, resposta antecipatória e recuperação;

$\#=p<0,05$, basal vs. recuperação, no grupo PM.

Dados apresentados como média \pm EPM. $C=$ grupo Controle; $\mathrm{PC}=$ grupo Praticante de Corrida;

PM = grupo Praticante de Musculação.

FIGURA 1 - Resposta da pressão arterial sistólica nos diferentes momentos do teste pressórico do frio.

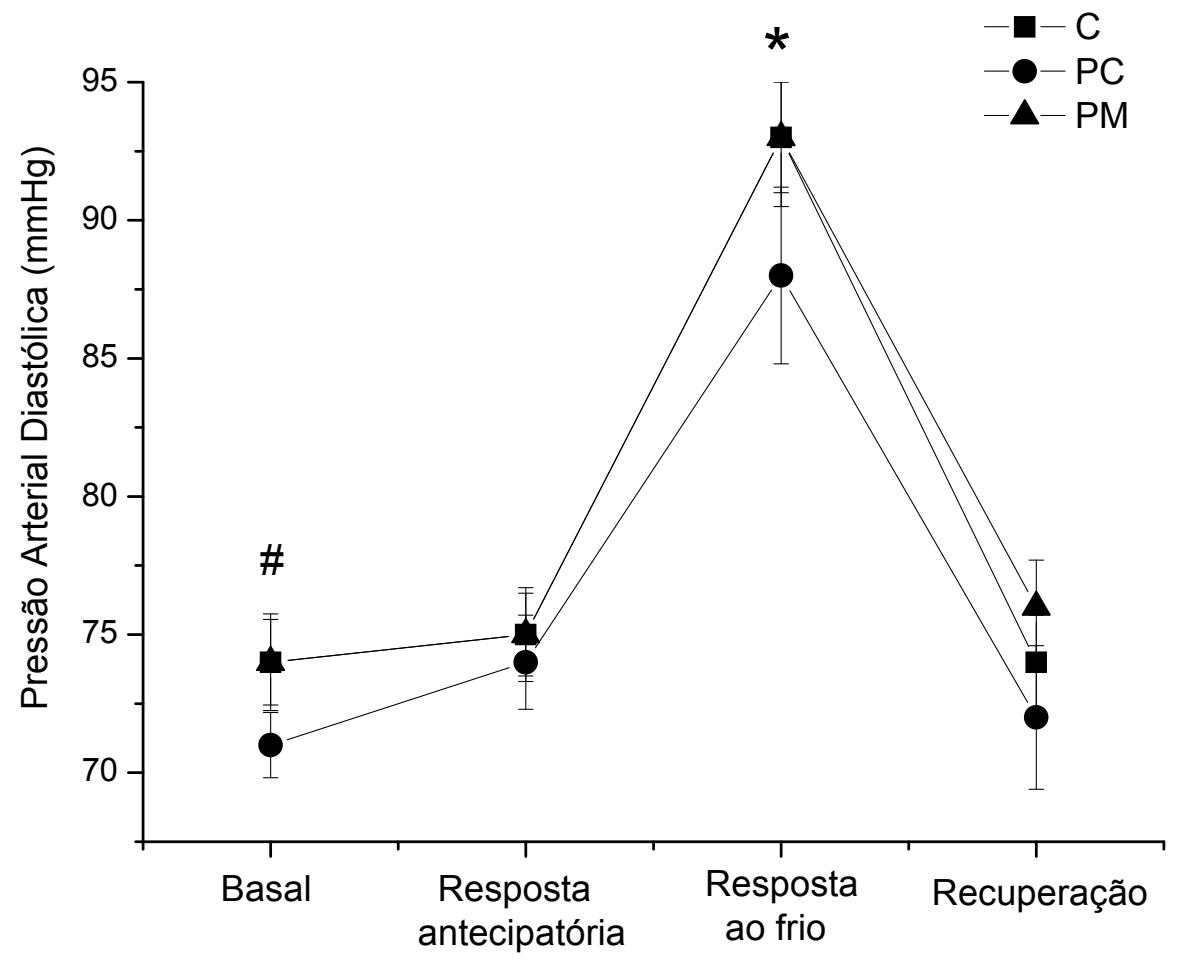

$\#=p<0,05, P C$ vs. $\mathrm{CePM}$;

${ }^{*}=p<0,05$, resposta ao frio vs. basal, resposta antecipatória e recuperação.

Dados apresentados como média \pm EPM. $C=$ grupo Controle; $\mathrm{PC}=$ grupo Praticante de Corrida;

PM = grupo Praticante de Musculação.

FIGURA 2 - Resposta da pressão arterial diastólica nos diferentes momentos do teste pressórico do frio.

Em relação à VFC (TABELA 2), o componente HF foi maior e os componentes LF e LF/HF foram menores para o grupo PC quando comparado ao grupo C. O grupo PM apresentou respostas intermediárias para esses componentes da VFC, de maneira que os valores não foram estatisticamente diferentes em comparação aos dois outros grupos. Não foram identificadas diferenças estatísticas entre os grupos para os parâmetros RMSSD e pNN50. 
TABELA 2 - Parâmetros da variabilidade da frequência cardíaca (VFC) nos grupos estudados.

${ }^{*}=p<0,05$, PC vs. C. Dados apresentados como média \pm EPM , seguidos pela mediana, apresentada entre parênteses.

$\mathrm{C}=$ grupo Controle;

$\mathrm{PC}=$ grupo Praticante de Corrida;

$\mathrm{PM}=$ grupo Praticante de Musculação. Os significados das siglas rMSSD, pNN50, HF e LF estão detalhados no tópico método.

$=p<0,05, P C$ vs. C; $\#=p<0,05, P C$ vs. PM; Comparações intra grupo:

$a=p<0,05$, repouso vs. demais momentos;

$b=p<0,05$, pico vs. 30 e 60 s da recuperação; $c=p<0,05,30$ s vs. 60 $s$ da recuperação. $\mathrm{C}=$ grupo Controle;

$\mathrm{PC}=$ grupo Praticante de Corrida;

PM - grupo Praticante de Musculação.

\begin{tabular}{lccccc}
\hline Grupos & rMSSD (ms) & pNN50 (\%) & HF (n.u) & LF (n.u) & LF/HF \\
\hline C (n=35) & $46,6 \pm 5,4(38,4)$ & $19,8 \pm 3,4(15,6)$ & $40,7 \pm 3,3(40,0)$ & $56,7 \pm 3,5(59,8)$ & $2,1 \pm 0,3(1,5)$ \\
PC (n= 28) & $44,4 \pm 2,5(39,7)$ & $21,2 \pm 2,6(17,7)$ & $55,1 \pm 4,0(56,6)^{*}$ & $43,1 \pm 4,0(42,2)^{*}$ & $1,1 \pm 0,2(0,7)^{*}$ \\
PM (n=31) & $52,3 \pm 5,7(45,9)$ & $26,5 \pm 4,0(24,7)$ & $45,8 \pm 3,6(42,6)$ & $52,4 \pm 3,6(57,0)$ & $1,53 \pm 0,2(1,3)$ \\
\hline
\end{tabular}

A FIGURA 3 mostra os valores da FCR, FC no (FC $30 s-$ pico; $\mathrm{PC}=-15 \pm 2 ; \mathrm{C}=-13 \pm 7 ; \mathrm{PM}=-10$ \pm 1 ; bpm). Entretanto, a recuperação da $\mathrm{FC}$ aos $60 \mathrm{~s}$ de recuperação (FC $60 \mathrm{~s}$ - pico) foi significativamente maior no grupo $\mathrm{PC}(-34 \pm 3 \mathrm{bpm})$ quando comparado aos grupos C (-24 $\pm 2 \mathrm{bpm})$ e PM (-23 $\pm 1 \mathrm{bpm})$. O delta da FC em $60 s$ por $30 s$ de recuperação (FC 60 $s-30$ s) também foi maior no grupo PC (-19 $\pm 2 \mathrm{bpm})$ quando comparado aos grupos $\mathrm{C}(-11 \pm 1 \mathrm{bpm})$ e $\mathrm{PM}$ $(-13 \pm 2 \mathrm{bpm})$. Independente da menor FC do grupo PC no pico do esforço, os resultados confirmam que ao final de um minuto de recuperação pós esforço máximo o grupo $\mathrm{PC}$ apresenta maior recuperação de FC. Por outro lado, o grupo PM não apresentou qualquer diferença em relação ao grupo $\mathrm{C}$.

Em relação ao incremento da $\mathrm{FC}$ (FC pico FCR), os grupos PC (126 $\pm 2 \mathrm{bpm}) \mathrm{C}(123 \pm 3$ bpm) e PM (119 \pm 3 bpm) não apresentaram diferença significativa, sugerindo FC de reserva similar nos três grupos. os grupos para o delta da FC em 30 s de recuperação

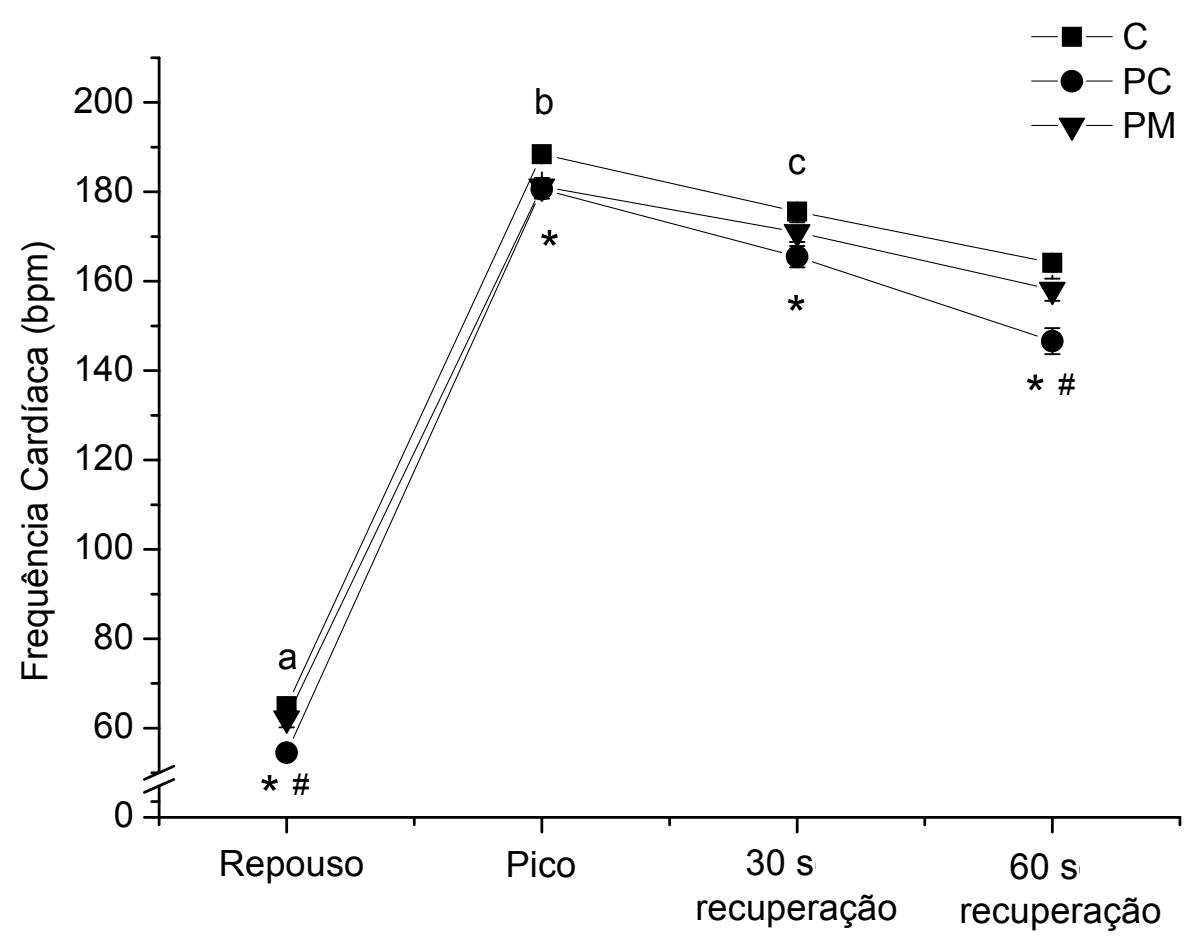

FIGURA 3 - Comportamento da frequência cardíaca em repouso, ao final do teste de esforço máximo e aos 30 e 60 segundos de recuperação dos grupos controle (C) e praticantes de corrida (PC) e musculação (PM). 


\section{Discussão}

O principal resultado do presente estudo é que, diferentemente da modulação do SNA induzida pela prática contínua de corrida de longa distância, a prática contínua em musculação, por longo prazo ( $\sim 10$ anos), não altera o SNA cardíaco quando comparado a pessoas não treinadas. Nossos resultados também ratificam o uso das técnicas indiretas FCR, FC de recuperação pós-teste ergométrico e VFC como clinicamente viáveis para avaliação do SNA.

Os parâmetros antropométricos e os valores de $\mathrm{VO}_{2 \max }$ confirmam que a estratificação dos grupos foi adequada. De fato, os maiores IMC, peso corporal e perimetrias do braço e perna com musculatura contraída encontrados no grupo PM já eram esperados por causa do maior volume muscular. Além do visual predomínio mesomórfico, a interpretação de maior volume muscular nesse grupo é corroborada pelos baixos valores das dobras cutâneas. Também já era esperado que o grupo PC apresentasse maior média para o $\mathrm{VO}_{2 \max }$, uma vez que é bem conhecido que a capacidade de captação e aproveitamento do oxigênio aumentam em consequência do treinamento em corrida de longa distância.

O grupo PC apresentou média de idade levemente superior. A expectativa inicial era obter grupos com idades estatisticamente iguais, mas, por se tratar de amostra de conveniência, não foi possível em virtude do perfil dos corredores. Em virtude das diferenças de idade, foram realizadas análises de covariância para minimizar possíveis interferências dessa variável.

No presente estudo, o grupo PC foi utilizado como controle positivo para modulação do SNA. De fato, já está bem estabelecido que o treinamento aeróbio promove alterações na modulação do SNA, em particular aumento da eferência parassimpática $^{5,8,10-12,16-17}$. A ausência de diferença estatística para FCR entre os grupos $\mathrm{PM} \mathrm{e} \mathrm{C}$ sugerem que a prática contínua de musculação por uma década não é suficiente para alterar o padrão modulatório do SNA. Essa interpretação é corroborada com os resultados da recuperação da FC pós teste de esforço máximo, que foi maior apenas no grupo PC (entre 40 a 46\%). De fato, sabe-se que tanto a FCR quanto a recuperação da FC pós teste de esforço, em particular no primeiro minuto da recuperação, são altamente influenciadas pelo tônus parassimpático ${ }^{3,8-11}$.

Sabe-se que maiores valores do componente HF e LF da VFC indicam maiores componentes parassimpático e simpático, respectivamente. Por sua vez, a razão LF/HF diminuída significa maior participação vagal ${ }^{12}$. Considerando que apenas o grupo PC apresentou maior HF e menores LF e $\mathrm{LF} / \mathrm{HF}$, quando comparado ao grupo $\mathrm{C}$, esses resultados fortalecem ainda mais a interpretação de que a prática de musculação por longo prazo não induz alterações significativas no SNA. Embora os índices no domínio do tempo (pNN50 e rMSSD) tenham sido determinados com a finalidade de se obter uma análise global da VFC, a validade dessas medidas são questionáveis para medidas inferiores a 20 min, o que poderia explicar porque não houve diferença entre os grupos pra essas medidas ${ }^{25,27}$.

Ainda não está completamente elucidado o mecanismo (ou mecanismos) pelo qual o treinamento aeróbio modula o SNA, mas parece envolver tanto o componente central quanto o periférico de regulação do $\mathrm{SNA}^{10}$. Acredita-se que o exercício possa agir de diferentes formas: Diretamente no centro regulador neural da $\mathrm{FC}^{10}$; no controle baroreflexo da FC via aumento do tônus vagal ${ }^{28}$; modulando metaborreceptores e mecanorreceptores dos músculos motores; facilitando a transmissão colinérgica vagal; e aumentando o antagonismo da atividade simpática via aumento da produção de óxido nítrico atrial ${ }^{8,10}$.

Em relação ao treinamento de força, os resultados são controversos. Estudos que avaliaram o SNA cardíaco por diferentes técnicas após treinamento de força de poucas semanas (quatro a oito semanas) mostram redução ${ }^{18}$ e aumento ${ }^{21}$ do tônus simpático, e ainda redução ${ }^{21}$ ou aumento ${ }^{18,20}$ do tônus parassimpático. Outros estudos não conseguiram encontrar qualquer efeito ${ }^{17,19-20}$.

Ressalta-se que não foram encontrados estudos que apresentassem efeitos de longo prazo do treinamento resistido isoladamente sobre o SNA, e seria pouco prudente comparar efeitos de curto prazo com efeitos de longo prazo. Nesse aspecto, ainda que nosso estudo seja transversal, ele traz contribuições importantes para a compreensão do impacto do treinamento contra resistência sobre o SNA.

Sabe-se que o exercício físico retira o organismo da homeostase, exigindo grandes ajustes do sistema cardiovascular pela via autonômica. Entretanto, é preciso, ponderar que as respostas cardiovasculares nas modalidades de corrida aeróbia e musculação não são as mesmas.

Em exercícios aeróbios se observa aumento do débito cardíaco em consequência das elevaçôes da FC e volume sistólico. Também se observa aumento 
da PAS, mas manutenção ou diminuição da PAD devido redução da resistência vascular periférica. $\mathrm{O}$ exercício aeróbio ainda promove resposta hipotensora pós exercício considerado de longa duração. Esse tipo de exercício mobiliza o SNA a partir, principalmente, do comando central e de mecanorreceptores ${ }^{2}$. Por sua vez, os exercícios contra resistência também promovem aumento da FC, mas com manutenção ou até redução do volume sistólico, causando apenas pequeno acréscimo do débito cardíaco. Em virtude do aumento da resistência vascular periférica causada pela obstrução mecânica do fluxo sanguíneo muscular, ocorre aumento da PAS e PAD. A resposta hipotensora pós exercício apresenta menor duração. Devido à grande produção muscular de metabólitos, a mobilização do SNA nesse tipo de exercício é bastante influenciada por quimiorreceptores musculares ${ }^{2}$.

De modo geral, em relação a eventos cardiovasculares, exercícios aeróbios produzem mais alterações relacionadas ao volume, enquanto exercícios contra resistência produzem mais alterações relacionadas à pressão ${ }^{12}$. É possível que uma ou mais dessas diferenças referentes aos ajustes cardiovasculares possa, quando em longo prazo, explicar o porquê de apenas exercícios aeróbios modularem o SNA.

As alteraçóes poderiam ocorrer no comando central, ou nas vias aferentes ou eferentes do SNA. Por exemplo, é bem conhecido que o exercício aeróbio modula a via aferente dos barorreceptores ${ }^{2,5,17,29}$, enquanto há sugestôes de que o treinamento resistido ou não altera ${ }^{19}$ ou até mesmo reduz ${ }^{17}$ o controle vagal do coração e a sensibilidade do barorreflexo. Ressalta-se que os pressorreceptores arteriais são o mais importante mecanismo de controle reflexo da $\mathrm{PA}$, e se caracterizam pela capacidade de se adaptarem a um novo limiar de funcionamento quando diante de alterações sustentadas ${ }^{1}$. É possível que as respostas cardiovasculares ao exercício aeróbio possam alterar o limiar de funcionamento de pressorreceptores, enquanto que as respostas cardiovasculares ao treinamento de musculação não teriam essa capacidade. Entretanto, são hipóteses que precisarão ser testadas.

É preciso ponderar que no presente estudo não foram realizados exames bioquímicos que permitissem avaliar o possível uso de drogas anabólicas. Recentemente foi mostrado que dose suprafisiológica $(-410 \mathrm{mg} / \mathrm{sem})$ de anabolizante pode reduzir o tônus parassimpático e aumentar o tônus simpático $^{30}$. Um estudo anterior havia mostrado resultado similar em modelo animal ${ }^{31}$. Nesse sentido, entendemos que o presente estudo contribui para a produção de conhecimento da área, mas não se pode deixar de destacar a necessidade de cautela nas interpretaçôes dos resultados.

Diferentemente das outras técnicas, o TPF não identificou diferenças do SNA entre os grupos. Outros autores também não conseguiram identificar alteração da resposta pressórica ao frio induzida pelo exercício físico ${ }^{32-33}$. Embora o TPF se mostre clinicamente importante na avaliação da função endotelial da artéria carótida ${ }^{34}$ e predição de eventos cardiovasculares em hipertensos ${ }^{4}$, é possível que o TPF não seja uma técnica suficientemente sensível para identificar pequenas alterações no SNA simpático de pessoas saudáveis.

De fato, a capacidade preditiva do TPF parece depender da presença de alguns fatores como doenças associadas (ex: doença arterial coronariana) ${ }^{35} \mathrm{e}$ idade avançada ${ }^{36-37}$. OTPF foi criado por Hines e BROWN ${ }^{38}$ para avaliar apenas o componente autonômico simpático ${ }^{15,36,39}$, e não o parassimpático. Embora, comparativamente a outros testes de estresse, o TPF seja validado e se destaque por minimizar os efeitos psicológicos ${ }^{15}$, deve-se reconhecer que esse teste gera dor que, por sua vez, aumenta a participação do SNA simpático $^{40}$. Sabe-se que dor é um parâmetro muito variável em humanos ${ }^{40} \mathrm{e}$, embora possivelmente tenha pouca influência em comparações do tipo "antes vs. depois", não podemos descartar a possibilidade dessa covariável influenciar na resposta do SNA de grupos independentes.

Diante disso, nossos achados corroboram o uso das técnicas indiretas FCR, FC de recuperação pós teste ergométrico e VFC como clinicamente viáveis para avaliação do SNA de pessoas sem doença associada, em particular enquanto estratégia de acompanhamento ${ }^{6}$. Por outro lado, não é possível fazer a mesma interpretação para o TPF.

Os resultados do presente estudo permitem concluir que a prática contínua em musculação por longo prazo, diferentemente da prática de corrida de longa distância, não é capaz de alterar significativamente o padrão modulatório do SNA. O conhecimento de que corridas aeróbias de longa distância e longo prazo, mas não a musculação, module o SNA, impacta diretamente na tomada de decisão referente à prescrição de exercício físico de pessoas com risco de doença cardiovascular. Entretanto, considerando as condiçōes metodológicas do presente estudo, em particular o fato de ser um estudo transversal, o qual não envolve intervenção e acompanhamento sistemáticos, é preciso prudência na extrapolação dos resultados. 


\begin{abstract}
Comparison of cardiovascular autonomic response among body builders, long distance runners and non-trained

The aim of the study was to compare the cardiovascular autonomic response (CAR) of recreational weight trainers, long distance runners and non-exercised subjects. Men, 21 to 55 years old, were grouped in: recreational weight trainers $(W, n=31)$, long distance runners $(R, n=28)$ and non-exercised $(C, n=35)$. Four strategies of evaluation of the CAR were selected: Resting heart rate (RHR), cold pressor test (CPT), heart rate variability (HRV) and heart rate recovery (HHR) following maximal exercise test. The RHR was lower $(R=54 \pm 2 ; W=62 \pm 2 ; C=65 \pm 2 b p m ;$ mean $\pm S E)$ and the HHR 60s post exercise was larger in the $R$ group $(R=34 \pm 3 ; W=23 \pm 1 ; C=24 \pm 2$ bpm). The $R$ group presented larger high-frequency (HF; $55.1 \pm 4.0$ n.u) and smaller low-frequency (LF; $43.1 \pm 4.0$ n.u) components of HRV than C group (HF $=40.7 \pm 3.3 ; \mathrm{LF}=56.7 \pm 3.5$ n.u.). The $\mathrm{W}$ group did not show any differences compared to $\mathrm{C}$ group. The study's conclusion was that long-term weight-training program, unlike of long-term running training, it is not able to alter significantly the regulatory pattern of CAR.
\end{abstract}

KEY wORDS: Strength training; Aerobic exercise; Parasympathetic nervous system; Sympathetic nervous system; Heart rate variability; Autonomic control of heart.

\title{
Referências
}

1. Irigoyen MC, Consolim-Colombo FM, Krieger EM. Controle cardiovascular: regulação reflexa e papel do sistema nervoso simpático. Rev Bras Hipertens. 2001;8:55-62.

2. Brum PC, Forjaz CLM, Tinucci T, Negrão CE. Adaptações agudas e crônicas do exercício físico no sistema cardiovascular. Rev Paul Educ Fís. 2004;18:21-31.

3. Lauer MS. Autonomic function and prognosis. Cleve Clin J Med. 2009;76:S18-22.

4. Nitenberg A, Chemla D, Antony I. Epicardial coronary artery constriction to cold pressor test is predictive of cardiovascular events in hypertensive patients with angiographically normal coronary arteries and without other major coronary risk factor. Atherosclerosis. 2004;173:115-23.

5. Hautala AJ, Kiviniemi AM, Tulppo MP. Individual responses to aerobic exercise: the role of the autonomic nervous system. Neurosci Biobehav Rev. 2009;33:107-15.

6. Nauman J, Janszky I, Vatten LJ, Wisløff U. Temporal changes in resting heart rate and deaths from ischemic heart disease. JAMA. 2011;306:2579-87.

7. César LAM. Frequência cardíaca e risco cardiovascular. Rev Assoc Méd Bras. 2007;53:456-9.

8. Wichi RB, Angelis K, Jones L, Irigoyen MC. A brief review of chronic exercise intervention to prevent autonomic nervous system changes during the aging process. Clinics. 2009;64:253-58.

9. Imai K, Sato H, Hori M, et al. Vagally mediated heart rate recovery after exercise is accelerated in athletes but blunted in patients with chronic heart failure. J Am Coll Cardiol. 1994;24:1529-35.

10. Coote JH. Recovery of heart rate following intense dynamic exercise. Exp Physiol. 2010;95:431-40.

11. Edwards KM, Wilson KL, Sadja J, Ziegler MG, Mills PJ. Effects on blood pressure and autonomic nervous system function of a 12-week exercise or exercise plus DASH-diet intervention in individuals with elevated blood pressure. Acta Physiol (Oxf). 2011;203:343-50.

12. Aubert AE, Steps B, Beckers F. Heart rate variability in athletes. Sports Med. 2003;33:889-919.

13. De Jong MMJ, Randall DC. Heart rate e variability analysis in the assessment of autonomic function in heart failure. J Cardiovasc Nurs. 2005;20:186-95.

14. Papaioannou VE. Heart rate variability, baroreflex function and heart rate turbulence: possible origin and implications. Hellenic J Cardiol. 2007;48:278-89.

15. Herkenhoff-Vieira FL, Lima EG. Testes de estresse laboratoriais e hipertensão arterial. Rev Bras Hipertens. 2007;14:98-103.

16. Mueller PJ. Exercise training attenuates increases in lumbar sympathetic nerve activity produced by stimulation of the rostral ventrolateral medulla. J Appl Physiol. 2007;102:803-13. 
17. Collier SR, Kanaley JA, Carhart Junior R, et al. Cardiac autonomic function and baroreflex changes following 4 weeks of resistance versus aerobic training in individuals with pre-hypertension. Acta Physiol (Oxf). 2009;195:339-48.

18. De Deus AP, De Oliveira CR, Simões RP, et al. Metabolic and cardiac autonomic effects of high-intensity resistance training protocol in Wistar rats. J Strength Cond Res. 2012;26:618-24.

19. Cooke WH, Carter JR. Strength training does not affect vagal-cardiac control or cardiovagal baroreflex sensitivity in young healthy subjects. Eur J Appl Physiol. 2005;93:719-25.

20. Heffernan KS, Christopher AF, Shinsako KK, Jae SY, Fernhall B. Heart rate recovery and heart rate complexity following resistance exercise training and detraining in young men. Am J Physiol Heart Circ Physiol. 2007;293:H3180-6.

21. Melo RC, Quitério RJ, Takahashi ACM, Silva E, Martins LEB, Catai AM. High eccentric strength training reduces heart rate variability in healthy older men. Br J Sports Med. 2008;42:59-63.

22. O'Brien E, Mee F, Atkins N, Thomas M. Evaluation of three devices for self-measurement of blood pressure according to the revised British Hypertension Society Protocol: The Omron HEM-705CP, Philips HP 5332, and Nissei DS-175. Blood Press Monit. 1996;1:55-61.

23. Dantas EM, Sant'anna ML, Andreão RV, et al. Spectral analysis of heart rate variability with the autoregressive method: What model order to choose? Comput Biol Med. 2012;42:164-70.

24. Malliani A, Pagani M, Lombardi F, Cerutti S. Cardiovascular neural regulation explored in the frequency domain. Circulation. 1991;84:482-92.

25. Malik M, Bigger JM, Camm AJ, et al. Heart rate variability: standards of measurement, physiological interpretation, and clinical use. Task Force of the European Society of Cardiology and the North American Society of Pacing and Electrophysiology. Eur J Heart. 1996;17:354-81.

26. Howley ET, Basset DR Jr, Welch HG. Criteria for maximal oxygen uptake: review and commentary. Med Sci Sports Exerc. 1995;27:1292-301.

27. Selig SE, Carey MF, Menzies DG, et al. Moderate-intensity resistance exercise training in patients with chronic heart failure improves strength, endurance, heart rate variability, and forearm blood flow. J Card Fail. 2004;10:21-30.

28. Liu JL, Kulakofsky J, Zucker IH. Exercise training enhances baroreflex control of heart rate by a vagal mechanism in rabbits with heart failure. J Appl Physiol. 2002;92:2403-8.

29. Brum PC, Bacurau AVN, Medeiros A, Ferreira JCB, Vanzelli AS, Negrão CE. Aerobic exercise training in heart failure: impact on sympathetic hyperactivity and cardiac and skeletal muscle function. Braz J Med Biol Res. 2011;44:827-35.

30. Maior AS, Carvalho AR, Marques-Neto SR, Menezes P, Soares PP, Nascimento JH. Cardiac autonomic dysfunction in anabolic steroid users. Scand J Med Sci Sports. 2012;18. [Epub ahead of print], DOI: 10.1111/j.16000838.2011.01436.x, 2012.

31. Pereira-Junior PP, Chaves EA, Costa-e-Sousa RH, Masuda MO, Carvalho ACC, Nascimento JHM. Cardiac autonomic dysfunction in rats chronically treated with anabolic steroid. Eur J Appl Physiol. 2006;96:487-94.

32. De Geus ECJ, Van Doornen LJP, Orlebeke JF. Regular exercise and aerobic fitness in relation to psychological make-up and physiological reactivity. Psychosom Med. 1993;55:347-63.

33. Bond Junior, Adams, Vaccaro, Blakely, Franks, Williams, Obisesan, Millis, 2001.

34. Kawano H, Tanimoto M, Yamamoto K, et al. Resistance training in men is associated with increased arterial stiffness and blood pressure but does not adversely affect endothelial function as measured by arterial reactivity to the cold pressor test. Exp Physiol. 2008;93:296-302.

35 Sevre K, Rostrup M. Blood pressure and heart rate responses to cold pressor test in patients admitted to hospital due to chest pain. Blood Press. 1999;8:110-13.

36. Menkes MS, Matthews KA, Krantz DS, et al. Cardiovascular reactivity to the cold pressor test as a predictor of hypertension. Hypertens. 1989;14:524-30.

37. Kasagi F, Akahoshi M, Shimaoka K. Relation between cold pressor test and development of hypertension based on 28-year follow-up. Hypertens. 1995;25:71-6.

38. Hines EA, Brown GE. The cold pressor test for measuring the reactibility of the blood pressure: data concerning 571 normal and hypertensive subjects. Am Heart J. 1936;11:1-9.

39. Victor VG, Leimbach Junior WN, Seals DR, Wallin BG, Mark AL. Effects of the cold pressor test on muscle sympathetic nerve activity in humans. Hypertens. 1987;9:429-36.

40. Hamunen K, Kontinen V, Hakala E, Talke P, Paloheimo M, Kalso E. Effect of pain on autonomic nervous system indices derived from photoplethysmography in healthy volunteers. Br J Anaesth. 2012;108:838-44, 2012. 


\section{Agradecimentos}

Agradecemos as agências de fomento CAPES e CNPq por recursos que permitiram a aquisição da estrutura necessária para a realização do projeto.

\begin{tabular}{r|l} 
ENDEREÇo & \\
Wellington Lunz & Recebido para publicação: 21/01/2013 \\
do Espírito Santo & Revisado: 16/05/2013 \\
ba - SP - BRASIL & Aceito: 18/07/2013 \\
&
\end{tabular}

